\title{
Spielplangestaltung im Kampf um Anerkennung - Diversifizierung als Legitimationsstrategie
}

\section{Bianca Michaels}

\section{Zusammenfassung}

Deutschlandweit lässt sich seit einigen Jahren eine kontinuierlich steigende Zahl an neuen Veranstaltungsformen und -formaten außerhalb etablierter Genre- und Spartenbezeichnungen an öffentlich getragenen Theatern beobachten. Besonders ausgeprägt ist diese Tendenz zur Diversifizierung in der Spielplangestaltung der Münchner Kammerspiele seit der Spielzeit 2015/2016. Ziel des Beitrags ist die Untersuchung des Zusammenhangs von Spielplangestaltung und Legitimationsdiskursen am Beispiel der Kontroverse um die Münchner Kammerspiele unter der Intendanz von Matthias Lilienthal 2018.

\section{Schlüsselwörter}

Legitimität $\bullet$ Legitimation $•$ Spielplangestaltung $\bullet$ Theaternahes

Rahmenprogramm • Münchner Kammerspiele

\section{$1 \quad$ Einleitung}

Open Border Kongress, Internationale Schlepper- und Schleusertagung, Welcome Café für Geflüchtete, diverse Stadtprojekte und Stadttheaterfestivals, Workshops, Diskursformate, ein Boxkampf usw. - die Liste von Veranstaltungen außerhalb etablierter Genre- und Spartenbezeichnungen eines Stadttheaters ließe sich anhand

\footnotetext{
B. Michaels $(\varangle)$

Department Kunstwissenschaften, Ludwig-Maximilians-Universität München, München, Deutschland

E-Mail: michaels@1mu.de
} 
der Spielpläne der Münchner Kammerspiele seit der Spielzeit 2015/2016 noch lange fortführen. Gleichzeitig sind vergleichbare Entwicklungen in den Spielplänen öffentlich getragener Theater auch deutschlandweit zu beobachten. Ziel des vorliegenden Beitrags ist die Untersuchung des Zusammenhangs von Legitimität und Spielplangestaltung öffentlich getragener Theater anhand aktueller Entwicklungen in der Diversifizierung der Veranstaltungsangebote.

\section{Diversifizierung der Programmplanung öffentlich getragener Theater in Deutschland}

Eine Analyse der durch den Deutschen Bühnenverein als Interessen- und Arbeitgeberverband der Theater und Orchester in Deutschland publizierten Theaterstatistik für die Spielzeit 2017/2018 zeigt deutlich, dass sich die öffentlich getragenen Theater hinsichtlich der von ihnen angebotenen Veranstaltungen in den vergangenen Jahren signifikant diversifiziert haben. Der Deutsche Bühnenverein hat darauf seit der Saison 2004/2005 mit der Einführung der neuen Rubrik „theaternahes Rahmenprogramm“ zusätzlich zur bereits bestehenden Kategorie „sonstige Veranstaltungen“ reagiert. Allein die Gesamtsumme der Veranstaltungen, welche in der Theaterstatistik unter den beiden Rubriken "sonstige Veranstaltungen“ und ,theaternahes Rahmenprogramm" gefasst werden, ${ }^{1}$ hat sich seit der Spielzeit 1991/1992 von insgesamt 6551 auf 23.191 Veranstaltungen in der Saison 2017/2018 fast vervierfacht (Deutscher Bühnenverein 1993, 2019). Es handelt sich bei den dort aufgeführten Veranstaltungen um verschiedene Formen wie szenische Lesungen, Performances, Konzerte, Workshops etc. Darüber hinaus sind zahlreiche neue Formen im Programmangebot zu verzeichnen, wie beispielsweise weitreichende Kulturvermittlungsangebote, Vortrags- und Diskursformate, partizipative Projekte und ortsspezifische Stückentwicklungen. ${ }^{2}$ Obgleich die hier angeführten Beispiele zeigen, dass die Bandbreite der Veranstaltungen hinsichtlich Ästhetik, Produktionsweisen, Zielpublikum etc. äußerst heterogen ist,

\footnotetext{
${ }^{1}$ Alltagssprachlich werden die hier genannten Veranstaltungen häufig als Veranstaltungen der „fünften Sparte“ bezeichnet.

${ }^{2}$ Wichtig ist in diesem Zusammenhang der Hinweis, dass die Meldung in der Statistik nachrichtlich durch die Theaterbetriebe an den Deutschen Bühnenverein erfolgt. Deshalb werden einige Veranstaltungen beispielsweise in Haus A unter der Rubrik ,,sonstige Veranstaltungen“ und in Haus B unter ,theaternahes Rahmenprogramm“ geführt. Obgleich der Bühnenverein eine deutliche Trennung vorsieht (Theaternahes Rahmenprogramm: kein oder geringes Eintrittsgeld), wird es - wie Erkundigungen im Feld gezeigt haben - nicht immer einheitlich eingetragen. Aus diesem Grund werden hier beide Rubriken zusammengefasst.
} 
lassen sie sich im vorliegenden Zusammenhang heuristisch als Veranstaltungen außerhalb des etablierten Repertoirebetriebs zusammenfassen.

$\mathrm{Zu}$ sehen ist in Abb. 1 ein signifikanter Anstieg, sodass deutschlandweit in der Spielzeit 2017/2018 insgesamt fast $30 \%$ aller Veranstaltungen an deutschen

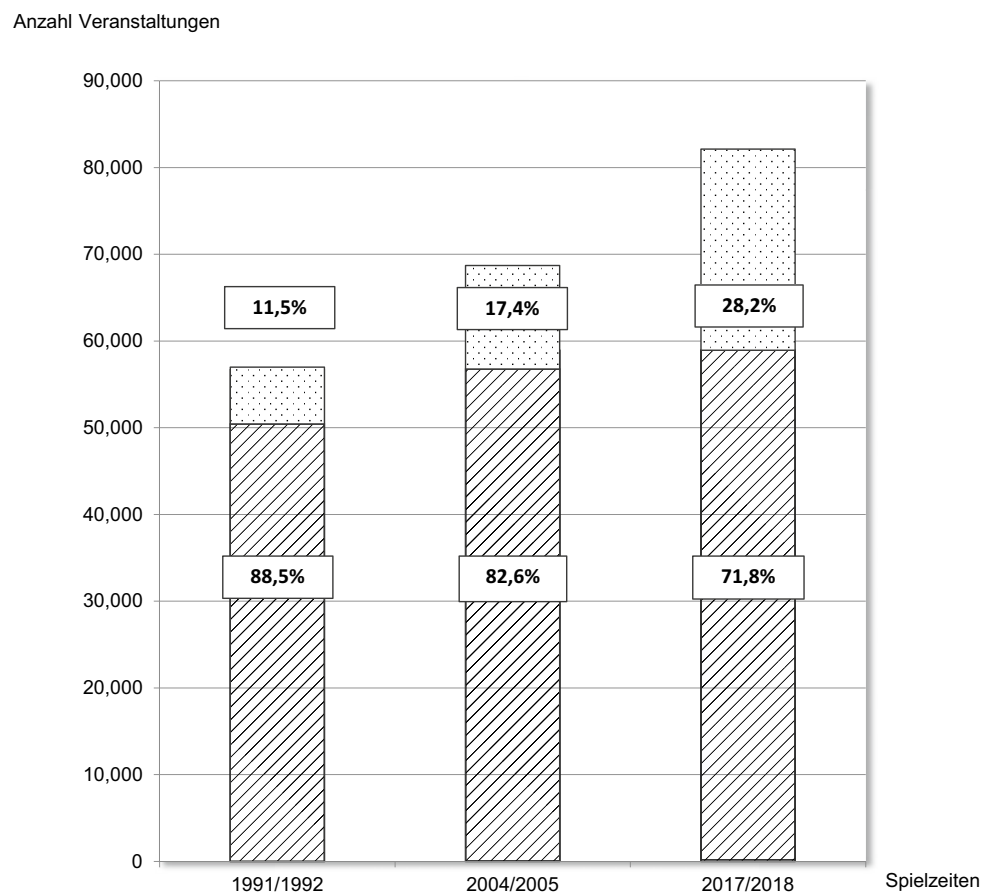

$\square$ Sonstige Veranstaltungen' und 'theaternahes Rahmenprogramm'

घVeranstaltungen der 'etablierten' Sparten (Oper, Schauspiel, Kinder- und Jugendtheater, Konzert etc.)

Abb. 1 Deutschlandweite Entwicklung seit der Spielzeit 1991/1992: „,Sonstige Veranstaltungen“ und ,theaternahes Rahmenprogramm“. (Quelle: Deutscher Bühnenverein 1993, 2006, 2019. (C) Bianca Michaels, 2020) 
Stadt- und Staatstheatern den ,sonstigen Veranstaltungen“ und dem ,theaternahen Rahmenprogramm“ zuzurechnen sind. ${ }^{3}$ Eine Analyse der Theaterstatistik des Deutschen Bühnenvereins für die Spielzeit 2017/2018 verdeutlicht darüber hinaus, dass über $91 \%$ der öffentlich getragenen Theater in der angegebenen Spielzeit Veranstaltungen der Kategorien ,theaternahes Rahmenprogramm“ und/oder „sonstige Veranstaltungen“ angeboten haben.

Dieses Bild eines fundamentalen institutionellen Wandels in der Programmgestaltung zeigt sich sowohl deutschlandweit wie auch an einzelnen Häusern. Ein besonders prägnantes Beispiel sind hier die Münchner Kammerspiele. Das kommunal getragene Theater eignet sich in besonderer Weise als Fallbeispiel, da signifikante Veränderungen in der Spielplangestaltung nicht nur mit dem Intendanzwechsel zur Spielzeit 2015/2016 zusammenfallen, sondern die Intendanz insbesondere in den Jahren 2016 und 2018 in der lokalen und überregionalen Medienberichterstattung und der kommunalen Kulturpolitik ausführlich diskutiert wurde. Aus diesem Umstand resultiert der hierzulande selten $\mathrm{zu}$ beobachtende Umstand, dass auch vonseiten zumindest einiger Vertreter*innen der Kulturpolitik dezidiert inhaltliche Äußerungen zur Programmgestaltung diskutiert wurden und dokumentiert sind.

\section{Fallbeispiel: Programmgestaltung Münchner Kammerspiele seit 2015}

Bereits während der vorhergegangenen Intendanzen von Frank Baumbauer (Spielzeit 2001/2002-2008/2009) und Johan Simons (Spielzeit 2010/2011-2014/2015) wiesen die Münchner Kammerspiele eine hohe Affinität zu neuen Formen und Formaten sowie zur Internationalisierung des Stadttheaters auf. ${ }^{4} \mathrm{Zu}$ nennen sind im vorliegenden Zusammenhang unter anderem zahlreiche von den Kammerspielen durchgeführte und wegweisende Stadtprojekte wie z. B. „Bunnyhill“ (2003)

\footnotetext{
${ }^{3}$ In der Theaterstatistik selbst werden die ergänzenden Angebote zur Vermittlung und zur Begleitung von Theaterveranstaltungen seit 2006, d. h. seit der Spielzeit 2004/2005 als Veranstaltungen im ,theaternahen Rahmenprogramm“ zwar separat ausgewiesen, jedoch nicht $\mathrm{zu}$ den aufsummierten Veranstaltungen aus den anderen Sparten hinzugerechnet.

${ }^{4}$ Auf ästhetische Schwerpunktsetzungen und Differenzierungen hinsichtlich ästhetischer Experimente innerhalb der verschiedenen Kategorien wird an dieser Stelle bewusst verzichtet, da der Fokus hier auf anderen Aspekten liegt.
} 


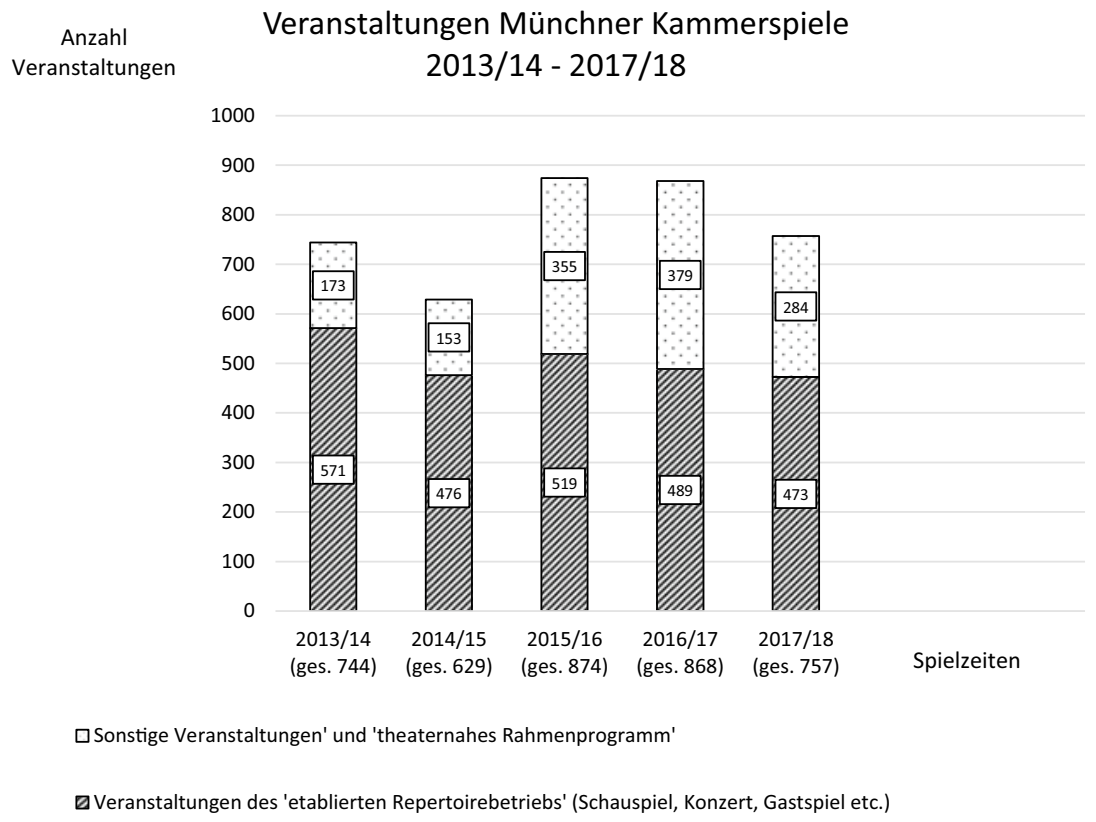

Abb. 2 Veranstaltungen Münchner Kammerspiele 2013/2014-2017/2018. (Quelle: Deutscher Bühnenverein 2015-2019. (C) Bianca Michaels, 2020)

und „Urban Prayers“ (2013). ${ }^{5}$ Unter der Intendanz von Matthias Lilienthal ab der Spielzeit 2015/2016 lässt sich ein sprunghafter Anstieg der ,sonstigen Veranstaltungen“ und des „theaternahen Rahmenprogramms“ von insgesamt 153 Veranstaltungen in der letzten Simons-Spielzeit 2014/2015 um mehr als das Doppelte auf insgesamt 355 Veranstaltungen dieser beiden Kategorien in der Spielzeit 2015/2016 beobachten. Prozentual steigt der Anteil der genannten Veranstaltungen von $23 \%$ bzw. $24 \%$ in den Spielzeiten 2013/2014 und 2014/2015 auf über $40 \%$ in den darauffolgenden zwei Spielzeiten unter der Leitung von Matthias Lilienthal (vgl. Abb. 2).

Insbesondere nach der ersten Spielzeit 2015/2016 unter der Intendanz von Matthias Lilienthal in München betont der 2017 veröffentlichte künstlerische

\footnotetext{
${ }^{5}$ Auf die Bedeutung des Förderfonds „Heimspiel“ der Kulturstiftung des Bundes für die Entwicklung von Stadtprojekten geht der Beitrag von Lukas Stempel (2020) in diesem Band ein.
} 
Geschäftsbericht des Theaters resümierend die ,tiefgreifenden ästhetischen und strukturellen Neuerungen“, um das Vorhaben umzusetzen, jüngere Zielgruppen an das Haus zu binden:

\begin{abstract}
„Die Kammerspiele haben mit Beginn der Spielzeit 2015/2016 inhaltliche und ästhetische Parameter, auf denen das Selbstverständnis eines Stadttheaters beruht, auf durchaus fundamentale Weise verschoben. [...] Matthias Lilienthal und sein Team möchten damit erreichen, dass das Theater nicht länger nur ein Ort der kulturellen Selbstbestimmung eines überwiegend deutschstämmigen Publikums bleibt, sondern auch für Zuschauerinnen und Zuschauer mit migrantischem Hintergrund zugänglich wird und damit der demographischen Entwicklung innerhalb der Stadt München und des Landes Bayern Rechnung trägt. [...] Eine Vielzahl an Diskussionen, Konzerten und thematischen Wochenenden festigen ihren Ruf als eine Institution, an der leidenschaftlich um die Grundfragen von Kunst und Gesellschaft gestritten wird“ (Eigenbetrieb Münchner Kammerspiele 2017, S. 2).
\end{abstract}

Was hier als leidenschaftlicher Streit um Grundfragen von Kunst und Gesellschaft bezeichnet wird, liest sich in der Medienberichterstattung seit 2016 folgendermaßen: Die dpa nennt das, was sich in München 2016 ereignet, einen „Theaterkrach“ und berichtet von einem „Debakel“ (dpa 2016), die Süddeutsche Zeitung von einem „Skandal“ (Dössel 2016a) und der Tagesspiegel titelt: „Theaterkrise an Bayerns bester Bühne: Münchner Kummerspiele“ (Guyton 2016). Anlass für die überregional in den Feuilletons geführten Diskussionen über eine Krise der Münchner Kammerspiele war u. a. die Ankündigung dreier Ensemblemitglieder, die Kammerspiele zum Ende der Spielzeit zu verlassen. Beispielsweise urteilt Christine Dössel in der Süddeutschen Zeitung: „Dass die Performeritis an dem Haus derart um sich greift, dass sie jegliches traditionellere Sprechtheater hinwegrafft, ist das eine. Dass sie keine großen, wichtigen, dem einstigen Niveau des Hauses würdigen Arbeiten hervorbringt, das andere. [...] das meiste ist Mittelmaß, harmlos, oberflächlich, simpel: Pipifax-Theater mit dem Anspruch, erklärend, belehrend und gerne auch migrationshintergründig sozial, global und politisch korrekt zu sein.“ (Dössel 2016b). Laut der Kritikerin der Süddeutschen Zeitung fehle „Lilienthals Sozialtheaterverein“ die Kunst (ebd).

Im Frühjahr 2018 folgt erneut eine intensive öffentliche Auseinandersetzung um die Münchner Kammerspiele - sowohl in den lokalen Medien wie auch in den lokalen kulturpolitischen Gremien: Der kulturpolitische Sprecher der CSUFraktion im Stadtrat, Richard Quaas kündigt im Frühjahr 2018 nicht nur an, dass die Zeit der finanziellen und künstlerischen Experimente an den Kammerspielen vorbei sei (Schleicher 2018), in einem Facebook-Post bezeichnet der Stadtrat 
das Programm der Kammerspiele unter der Intendanz Lilienthal als Firlefanz ${ }^{6}$. Am 20. März 2018 äußert sich der Politiker dezidiert zu seiner Auffassung eines Stadttheaters: Das Theater der Stadt habe ,andere Aufgaben als eine x-beliebige Experimentierbühne zu sein“ (Quaas 2018; Leucht 2018). Es folgen eine öffentliche Demonstration unter dem Titel „Kammer! Jetzt!“ in München, im Rahmen derer der kulturpolitische Diskurs über die Kammerspiele diskutiert werden sollte (o. A. 2018), eine Sondersitzung des Kulturausschusses des Münchner Stadtrats, offene Briefe und Unterschriftenlisten sowie unzählige Kommentare auf diversen Social Media Kanälen. Obgleich Veranstaltungen außerhalb des etablierten Repertoirebetriebs weder von den lokalen Medien noch vom kulturpolitischen Sprecher der Münchner CSU-Fraktion explizit benannt werden, verweisen die in der öffentlichen Debatte verwendeten Begrifflichkeiten wie „Lilienthals Sozialtheaterverein“, „Pipifax-Theater“ und „Firlefanz“ in Abgrenzung zu den so genannten großen und wichtigen Arbeiten deutlich darauf, dass hier gerade auch die schwer in einem Begriff zu fassenden Formen und Formate des theaternahen Rahmenprogramms und der sonstigen Veranstaltungen Anlass zur Kritik geben.

Nachdem sich die CSU-Fraktion des Münchner Stadtrats gegen eine Verlängerung des Vertrags von Matthias Lilienthal über das Jahr 2020 hinaus ausgesprochen hat, verkündet der Intendant Mitte März 2018, in München sei kein Rückhalt für eine Verlängerung seiner Arbeit gewährleistet, nachdem die CSU-Fraktion im Münchner Stadtrat bereits zwei Wochen zuvor einen Beschluss gegen eine Vertragsverlängerung gefasst habe (Höbel 2018). Ohne die Zustimmung der CSU-Stadträte galt es aufgrund der Zusammensetzung des Münchner Stadtrats zu diesem Zeitpunkt als unwahrscheinlich, dass sich eine Mehrheit für eine weitere Amtszeit Lilienthals als Intendant finden würde.

Die öffentliche Debatte kann an dieser Stelle nicht detailliert wiedergegeben und analysiert werden. Stattdessen werden im Folgenden anhand einiger wesentlicher Diskursstränge der öffentlichen Debatte Rückschlüsse auf den politisch und medial geführten ,Kampf um Anerkennung' zu ziehen sein: Was gilt als legitim in Bezug auf die Spielplangestaltung des Hauses - und was nicht? Fasst man im Anschluss an neoinstitutionalistische Organisationstheorien Legitimität als eine grundlegende Bedingung für die Existenz von Institutionen auf, so lässt sich auch im Hinblick auf Theater und insbesondere vor dem Hintergrund der Bedeutung öffentlicher Förderung im deutschen Theatersystem im Anschluss an die prägnante Formulierung des Soziologen W. Richard Scott konstatieren: „Like some

\footnotetext{
${ }^{6}$ Der Post ebenso wie der gesamte Facebook-Account „Stadtrat.Richard.Quaas“ des 2020 aus dem Münchner Stadtrat ausgeschiedenen kulturpolitischen Sprechers der CSU-Fraktion ist zwischenzeitlich gelöscht worden.
} 
other invisible properties such as oxygen, the importance of legitimacy become immediately and painfully apparent only if lost, suggesting that it is not a specific resource, but a fundamental condition of social existence" (Scott 2014, S. 72). Vor diesem Hintergrund ist zu fragen, ob die hier skizzierten öffentlichen Debatten um die Münchner Kammerspiele möglicherweise als Indikator eines sich vollziehenden Delegitimationsprozesses des öffentlich getragenen Theaters zu interpretieren sind.

\section{$4 \quad$ Legitimität und Legitimation}

Um die Frage nach einem sich möglicherweise vollziehenden Delegitimationsprozess zu klären, ist zunächst zu differenzieren, um welche Art von Legitimität es sich handelt. Der Begriff der Legitimität bedeutet im politisch-gesellschaftlichen Zusammenhang zunächst ganz allgemein das Vertrauen in die Rechtmäßigkeit einer bestehenden politischen Herrschaft oder sozialen Ordnung (Dammayr et al. 2015, S. 9). Die Sozialwissenschaften begreifen Legitimität als Rechtmäßigkeit eines politischen Systems und Legitimation als Verfahren zur Herstellung von Legitimität (Westle 2000, S. 341). Für das oben skizzierte Fallbeispiel der Kammerspiele könnte man folglich davon ausgehen, dass sowohl in der lokalen Medienberichterstattung wie auch in Teilen der CSU-Stadtratsfraktion die Legitimität des Schauspielhauses abnimmt bzw. dass die oben geschilderten Auseinandersetzungen deutliche Indizien für eine schwindende Legitimität in Politik und medialer Fachöffentlichkeit der Kammerspiele München sind.

Um diesen Zusammenhang näher zu beleuchten, wird im Folgenden das bereits 1965 durch den Politikwissenschaftler David Easton entwickelte und später weiter ausgearbeitete Modell politischer Unterstützung mit seiner Differenzierung von spezifischer und diffuser Legitimität sozialer Ordnung herangezogen. Easton beschreibt den Begriff Unterstützung (,,support“) ,as an attitude by which a person orients himself to an object either favorably or unfavorably“ (Easton 1975, S. 436). Hintergrund des Modells ist die Feststellung, dass politische Unzufriedenheit nicht immer, oder gar üblicherweise, ein Signal für politischen Wandel darstellt. Daher genüge es nicht, lediglich zu konstatieren, die politische Unterstützung beispielsweise für politische Instanzen sei zu einem bestimmten Zeitpunkt auf niedrigem Level oder Intensität bzw. von kurzer Dauer. Deshalb führt Easton die Unterscheidung zwischen spezifischer und diffuser Unterstützung ein, deren Gewinn darin liegt, dass diese zwei Arten von Unterstützung jeweils unabhängig voneinander variieren können (ebd., S. 344). Dabei ist die spezifische Unterstützung objekt-spezifisch, d. h. sie bezieht sich auf wahrgenommene 
Entscheidungen, Äußerungen, Handlungen oder den generellen Handlungsstil der betreffenden Behörden und der jeweils bevollmächtigten Akteure (,,authorities“), die in einem direkten Zusammenhang mit den Akteuren stehen (ebd., S. 437 f.). Als ,authorities“ bezeichnet Easton hierbei alle Behörden und in der Öffentlichkeit stehende Amtsinhaber*innen von Gesetzgeber*innen, Richter*innen bis hin zu höheren städtischen Beamt*innen wie auch deren jeweilige Institutionen. Wichtig ist in diesem Zusammenhang die Erweiterung, mit der er vorschlägt, nicht nur explizite Aktionen der Akteure in das Konzept zu integrieren, sondern auch ,their perceived general performance“ (ebd., S. 438). Im Kontext der , wahrgenommenen Performance' wird deutlich, dass es hier auch um die Frage geht, ob die jeweiligen Mitglieder eines politischen Systems die Erwartungen an die generelle Performance der jeweiligen Behörden als erfüllt betrachten, d. h. ,that the members perceive, whether correctly in some objective sense or not, that the fulfilment of their needs and demands can be associated with the authorities in some way“ (ebd., S. 438). Im vorliegenden Fall geht es somit um die Frage, ob die künstlerische Leitung die Erwartungen der kulturpolitischen Entscheidungsträger*innen und der Fachpresse an die generelle Performance der Münchner Kammerspiele als Eigenbetrieb der Stadt München erfüllt.

In Abgrenzung zur spezifischen Unterstützung fasst Easton den Begriff der diffusen Unterstützung folgendermaßen zusammen: „It refers to evaluations of what an object is or represents - to the general meaning it has for a person - not of what it does" (Easton 1975, S. 444). Hier werden auch Outputs toleriert, denen Personen negativ gegenüberstehen. Diffuse Unterstützung ist folglich normalerweise von längerer Dauer und weniger volatil als spezifische Unterstützung (ebd.). Somit betrifft spezifische Unterstützung vor allem einzelne Amtsinhaber*innen und Führungspersonen, während sich die diffuse Unterstützung eher an die Institutionen und Organisationen selbst richtet. Dies kann im vorliegenden Fall die Kammerspiele als Theater der Stadt München betreffen oder die Einrichtung von öffentlich getragenem Theater als Institution. Die Unterscheidung liegt dort, wo es um fundamentale Belange geht (diffuse Unterstützung) oder mehr um das, was lokale Autoritäten tun oder nicht tun (spezifische Unterstützung). Diese jeweiligen Unterstützungsperspektiven sind für den Gegenstand des Theaters vor allem deshalb fruchtbar, weil sie es ermöglichen, in der Analyse zwischen der Legitimität des Theaters als Institution, eines spezifischen Theaters (wie hier der Kammerspiele München) und einer spezifischen Theaterleitung (in diesem Fall Matthias Lilienthal) zu differenzieren. 


\section{$5 \quad$ Spezifische und diffuse Legitimation am Beispiel der Münchner Kammerspiele}

David Easton definiert in seinem Konzept politischer Unterstützung Legitimität als Übereinstimmung der eigenen Werte und Vorstellungen mit den Objekten des politischen Systems. Obgleich das Konzept im Hinblick auf politische Systeme erarbeitet wurde, eignet es sich bzgl. seiner Unterscheidung in spezifische und diffuse Arten von Unterstützung, um auf den hier betrachteten Gegenstand der Theater übertragen zu werden. Im Hinblick auf die Münchner Kammerspiele sind die oben dargestellten Ereignisse folgendermaßen zu interpretieren:

Die CSU-Fraktion im Münchner Stadtrat bzw. die Mehrheit der Fraktion sah offenbar die spezifische Anerkennungswürdigkeit der Intendanz Lilienthals im Frühjahr 2018 nach zweieinhalb Spielzeiten als nicht (mehr) gegeben an. Die oben zitierten Äußerungen des kulturpolitischen Sprechers und der gefasste Beschluss der Fraktion lassen hier keine andere Folgerung zu.

Allerdings legen die veröffentlichten Äußerungen nahe, dass sich die Wahrnehmung einer krisenhaften Situation der Kammerspiele durch die CSUStadtratsfraktion in erster Linie als Entzug der spezifischen Unterstützung der für das Theater künstlerisch verantwortlichen Akteure und hier in erster Linie des künstlerischen Leiters Matthias Lilienthal manifestiert. Legitimität für eine spezifische Intendanz, über deren Vertragsverlängerung im Stadtrat entschieden wird, ist vorhanden, wenn es den Akteuren gelingt, bei ihren Stakeholdern (Politik, Publikum, Medien) die Überzeugung zu schaffen und zu erhalten, dass die bestehende Intendanz mit ihrem jeweiligen künstlerischen Output für die betreffende Gesellschaft die bestmögliche ist. In den ersten zweieinhalb Jahren seiner Intendanz ist es Matthias Lilienthal mit seinem Team offensichtlich nicht gelungen, diese Überzeugung im Kulturausschuss des Stadtrats bzw. bei den Vertreter*innen der CSU und den Kritiker*innen des lokalen Feuilletons der Süddeutschen Zeitung zu schaffen.

Bemerkenswert ist in diesem Zusammenhang, dass die Legitimität der Münchner Kammerspiele selbst als öffentlich getragene Theaterorganisation im Rahmen der Debatte keineswegs infrage gestellt wird. Die diffuse Unterstützung der kulturpolitischen und journalistischen Akteure für die Kammerspiele als Organisation und das Theater als Institution erscheint nach wie vor hoch, während die sehr viel volatilere spezifische Unterstützung für die aktuelle künstlerische Ausrichtung unter der Intendanz von Matthias Lilienthal als gering zu bezeichnen ist. Dies legen sowohl die Äußerungen aus der Politik wie auch des lokalen Feuilletons in ihrer Argumentation nahe, dass das Theater quasi geschützt werden müsse vor der Intendanz Lilienthal: „Es besteht die Gefahr, dass hier ein Theater an die 
Wand gefahren wird. Nicht irgendein Theater, sondern eines der besten." (Dössel 2016b) und die Aussage des Stadtrats Quaas, die Kammerspiele seien „eines der ersten Häuser in Deutschland in der großen Tradition der Sprechtheater. Das ist unter Lilienthal verloren gegangen“ (Effern et al. 2018). Die Aussagen zu den Kammerspielen legen den Schluss nahe, dass hier das Theater selbst als eine Institution begriffen wird, die vor dem Niedergang bewahrt werden müsse. Mit dem Vokabular von Easton ausgedrückt: Die spezifische Übereinstimmung mit der aktuellen Performanz des Münchner Theaters ist zwar verhältnismäßig niedrig, die diffuse Unterstützung für das Haus jedoch scheint ungebrochen und stabil zu sein.

Die Unterscheidung in spezifische und diffuse Unterstützung verdeutlicht, warum die von den Medien als Krise bezeichnete Situation keine Krise für die Kammerspiele selbst als Organisation darstellt, da die prinzipielle Legitimität des Hauses nicht infrage gestellt wird. Ein grundsätzlicher Delegitimationsprozess in Bezug auf die kommunal getragenen Münchner Kammerspiele ist daraus folglich nicht abzuleiten. Gleichwohl sind die Dimensionen der Theaterleitung, der spezifischen Theaterorganisation der Münchner Kammerspiele und von Theater als Institution eng miteinander verwoben, wenn anhand der Person Matthias Lilienthal und dessen künstlerischer Entscheidungen auch öffentlich ausgehandelt und diskutiert wird, welche Funktionen die Kammerspiele als kommunales Theater für die Stadtgesellschaft in München bisher hatten und künftig möglicherweise einnehmen sollen.

\section{Spielplangestaltung als Arbeit an der Legitimation}

Die Tatsache, dass einige Akteure aus Politik und Medien ihre Erwartungen an die Kammerspiele als nicht erfüllt sehen, ist selbstverständlich nicht ausschließlich auf die Spielplangestaltung zurückzuführen, da sich Erwartungen an ein Theater auch auf Fragen des Repertoires, Besetzungen, Ästhetik, Gastspiele, Kommunikation etc. beziehen. ${ }^{7}$ Die Tatsache jedoch, dass die Vielzahl von Veranstaltungen außerhalb des etablierten Repertoires unter der Intendanz von Matthias Lilienthal ein Phänomen ist, das sich auch als deutschlandweite Erscheinung in der Programmplanung zeigt, lässt das Fallbeispiel als gewinnbringend für eine Übertragung auf die Theaterlandschaft in Deutschland mit ihren rund 140 öffentlich

\footnotetext{
${ }^{7}$ Nicht $\mathrm{zu}$ vergessen ist an dieser Stelle, dass die Herausforderung verfestigter Erwartungsstrukturen wiederum auch selbst Teil der Erwartungen ist, die einer neuen Intendanz entgegengebracht werden.
} 
getragenen Häusern erscheinen. Der oben skizzierte deutschlandweite Wandel in der Spielplangestaltung mit einem mittlerweile schon mehrere Jahre anhaltenden signifikanten Anstieg von „sonstigen Veranstaltungen“ und Veranstaltungen im „theaternahen Rahmenprogramm“ lässt sich als Antwort auf bereits bestehende Legitimationsdefizite und als Ausdruck eines größer zu fassenden Transformationsprozesses interpretieren. In diesem Prozess um Theater als Institution wird verhandelt, welche Ansprüche an Theater gestellt werden bzw. welche Ansprüche Theater bzw. deren Akteure an sich selbst stellen hinsichtlich der Fragen, wie mit den Spannungsfeldern zwischen verschiedenen institutionellen Logiken (Michaels 2021) und Legitimationsnarrativen umzugehen ist, welche Funktionen Theaterorganisationen in den jeweiligen Stadtgesellschaften wahrnehmen und was künftig als sogenanntes ,Kerngeschäft‘ des Theaters gilt. Damit wird auch neu verhandelt, was unter Theaterschaffenden, Publikum, Kritik und Politik jeweils als gesellschaftlich relevantes Theater gilt und durch welche Veranstaltungen sich öffentlich getragenes Theater legitimieren kann bzw. muss. Obwohl Veranstaltungen im „theaternahen Rahmenprogramm“ bereits seit der Spielzeit 2004/2005 als Kategorie in die Theaterstatistik des Deutschen Bühnenvereins aufgenommen werden, werden die dort aufgeführten Fallzahlen bisher nicht zur Gesamtzahl der Veranstaltungen hinzugezählt. Möglicherweise bewirkt ein weiteres Anhalten der oben beschriebenen Tendenz in der Spielplangestaltung öffentlich getragener Theater künftig neben einer erneuten Verschiebung der Kategorienbildung bzw. der Berechnungsgrundlage in der Theaterstatistik auch eine Verschiebung hinsichtlich der Erwartungsstrukturen an Theater und der in der Öffentlichkeit diskutierten Legitimationsnarrative. Matthias Lilienthal beschreibt in einem Interview mit der Neuen Zürcher Zeitung im Dezember 2016 den Zusammenhang zwischen gesellschaftlichem Wandel und dem sich derzeit vollziehenden Wandel am Theater folgendermaßen: „Die Auseinandersetzungen in alten Rechts-Links-Schemata funktionieren überhaupt nicht mehr, es gibt ein Patchwork von Identitäten, die sich frei kombinieren. Die Gesellschaft ist gerade dabei völlig neue Regeln für sich zu entwickeln. Und in dem Moment muss auch Theater seine Funktion neu definieren" (Noack 2016).

Indem die Spielplangestaltung das künstlerische Profil eines Theaters abbildet, lässt sich diese als sichtbare und auch jenseits des transitorischen Moments der Aufführung beobachtbare Manifestation des eigenen Selbstbildes als Organisation fassen, welche die jeweils geltenden vieldimensionalen Ansprüche an Theater und der Theater an sich selbst zum Vorschein bringt. Wenn Legitimation auch den Prozess der Rechtfertigung eigener Handlungen oder Handlungsergebnisse umfasst, lässt sich die Spielplangestaltung eines Theaters/einer künstlerischen 
Leitung als Arbeit an der eigenen Legitimation begreifen. ${ }^{8}$ Somit ist sie nicht nur als künstlerische Setzung und ästhetisches Programm zu verstehen, sondern zugleich als Argumentationsgrundlage und Legitimationsstrategie im ,Kampf um Anerkennung'. Die Programmplanung kann ein wesentliches Moment im Rahmen der Legitimationsstrategie sein bzw. gleichzeitig einer der Prüfsteine bei der Frage nach der Legitimität wie beispielsweise einer Intendanzverlängerung. Die hier geschilderte Kontroverse um die Münchner Kammerspiele vor dem Hintergrund der deutschlandweiten Entwicklungen von Veranstaltungen außerhalb des etablierten Repertoirebetriebs zeigt einen Ausschnitt der derzeit geltenden bzw. um Anerkennung ringenden Legitimationsnarrative. Sie macht dabei exemplarisch einige der Spannungsfelder sichtbar, die sich durch die unterschiedlichen und sich teilweise widersprechenden Erwartungshaltungen an ein öffentlich getragenes Theater ergeben.

\section{$7 \quad$ Nachtrag}

Die Münchner Kammerspiele werden nach Ende der Spielzeit 2017/2018 in der Kritikerumfrage der Fachzeitschrift „Theater Heute“ fast zum „Theater des Jahres" gewählt. In den beiden darauffolgenden Jahren 2019 und 2020 wird das Münchner Haus schließlich zwei Mal in Folge zum „Theater des Jahres“ gewählt (2019 neben weiteren Auszeichnungen wie „Inszenierung des Jahres“, „Schauspieler des Jahres“, „Nachwuchsschauspielerin des Jahres“, „Bühnenbild des Jahres“" etc.). ${ }^{9}$ Darüber hinaus erfährt das Haus national und international Anerkennung durch zahlreiche Einladungen zum Theatertreffen in Berlin sowie

\footnotetext{
${ }^{8}$ Zum Zusammenhang von Legitimität und Spielplangestaltung schreibt beispielsweise Friederike von Cossel: „Die Entwicklung und Gestaltung des Spielplans stellt [...] eine Organisationsaktivität dar, die institutionellen Anforderungen entsprechen muss, um eine größtmögliche Legitimität zu erlangen." (Von Cossel 2011, S. 37).

${ }^{9}$ In diesem Kontext ist zu fragen, welche Rolle die kulturpolitischen Kontroversen 2018 bei der Juryentscheidung gespielt haben und ob der Rückgang der Veranstaltungen außerhalb des etablierten Repertoirebetriebs in der Spielzeit 2017/2018 im Vergleich zu den ersten beiden Spielzeiten unter Lilienthal (vgl. Abb. 2) hier eine Rolle gespielt haben könnte. Im Rahmen dieses Beitrags und auf Basis der bislang vorliegenden Daten ist diese Frage, ob die Kammerspiele u. a. gerade wegen ihres auch 2017/2018 noch verhältnismäßig hohen Anteils an ,sonstigen Veranstaltungen“ und Veranstaltungen des ,theaternahen Rahmenprogramms“ $(37,5 \%)$ von der überregionalen Kritik positiv bewertet und fast zum „Theater des Jahres“ gewählt wurde oder vielmehr trotz ihres im deutschlandweiten Vergleich verhältnismäßig großen Anteils von Veranstaltungen außerhalb des etablierten Repertoirebetriebs, vorerst nicht $\mathrm{zu}$ beantworten.
} 
zu internationalen Gastspielen. Den Kampf um die Anerkennung der überregionalen Fachöffentlichkeit haben die Münchner Kammerspiele unter der Intendanz von Matthias Lilienthal offenbar deutlich für sich entscheiden können.

\section{Literatur}

Dammayr, M., D. Graß und B. Rothmüller. 2015. Legitimität und Legitimierung in der sozialwissenschaftlichen Debatte: eine Einführung in Theorien der Rechtfertigung und Kritik von Herrschaft. In Legitimität. Gesellschaftliche, politische und wissenschaftliche Bruchlinien der -Rechtfertigung dies, Hrsg. Dammayr, M., D. Graß und B. Rothmüller, 7-24. Bielefeld: Transcript.

Deutscher Bühnenverein. 1993. Theaterstatistik 1991/92. Die wichtigsten Wirtschaftsdaten der Theater, Orchester und Festspiele. Köln: Deutscher Bühnenverein.

Deutscher Bühnenverein. 2006. Theaterstatistik 2004/2005: Die wichtigsten Wirtschaftsdaten der Theater, Orchester und Festspiele. Köln: Deutscher Bühnenverein.

Deutscher Bühnenverein. 2015. Theaterstatistik 2013/14: Die wichtigsten Wirtschaftsdaten der Theater, Orchester und Festspiele. Köln: Deutscher Bühnenverein.

Deutscher Bühnenverein. 2016. Theaterstatistik 2014/2015. Die wichtigsten Wirtschaftsdaten der Theater, Orchester und Festspiele. Köln: Deutscher Bühnenverein.

Deutscher Bühnenverein. 2017. Theaterstatistik 2015/2016: Die wichtigsten Wirtschaftsdaten der Theater, Orchester und Festspiele. Köln: Deutscher Bühnenverein.

Deutscher Bühnenverein. 2018. Theaterstatistik 2016/2017: Die wichtigsten Wirtschaftsdaten der Theater, Orchester und Festspiele. Köln: Deutscher Bühnenverein.

Deutscher Bühnenverein. 2019. Theaterstatistik 2017/2018: Die wichtigsten Wirtschaftsdaten der Theater, Orchester und Festspiele. Köln: Deutscher Bühnenverein.

Dössel, C. 2016a. Als ließe der FC Bayern Lewandowski auf der Ersatzbank hocken. Süddeutsche Zeitung 3. November 2016. https://www.sueddeutsche.de/kultur/theater-als-liesseder-fc-bayern-lewandowski-auf-der-ersatzbank-hocken-1.3231419. Zugegriffen: 17 Juni 2020.

Dössel, C. 2016b. Theaterkrise in München. Kammerspiele? Jammerspiele! Süddeutsche Zeitung 11. November 2016. https://www.sueddeutsche.de/kultur/theaterkrise-in-muenchenkammerspiele-jammerspiele-1.3243228-0\#seite-2. Zugegriffen: 17 Juni 2020.

DPA. 2016. Theaterkrach: Brigitte Hobmeier kündigt Münchner Kammerspielen. Die Welt 3. November 2016. https://www.welt.de/regionales/bayern/article159244915/Brigitte-Hob meier-kuendigt-Muenchner-Kammerspielen.html. Zugegriffen: 17. Juni 2020.

Easton, David. 1975. A Re-Assessment of the Concept of Political Support. British Journal of Political Science 5 (4): 435-457.

Effern H., S. Hermanski und M. Zirnstein. 2018. Matthias Lilienthal hört als Intendant der Münchner Kammerspiele auf. Süddeutsche Zeitung 19. März 2018. https://www.sue ddeutsche.de/muenchen/kultur-in-muenchen-matthias-lilienthal-hoert-als-intendant-dermuenchner-kammerspiele-auf-1.3913360. Zugegriffen: 17. Juni 2020.

Eigenbetrieb Münchner Kammerspiele. 2017. Jahresabschluss für das Wirtschaftsjahr 2015/16. Lagebericht. muenchen-transparent.de, https://www.muenchen-transparent.de/ dokumente/4500227. Zugegriffen: 17 Juni 2020. 
Guyton, S. 2016. Theaterkrise an Bayerns bester Bühne. Münchner Kummerspiele. Tagesspiegel 21. November 2016. https://www.tagesspiegel.de/kultur/theaterkrise-an-bayernsbester-buehne-muenchner-kummerspiele/14873496.html. Zugegriffen: 17. Juni 2020.

Höbel, W. 2018. Angefeindeter Theaterchef Matthias Lilienthal kapituliert. In: Der Spiegel 19. März 2018. https://www.spiegel.de/kultur/gesellschaft/muenchen-theaterchef-mat thias-lilienthal-kapituliert-a-1198896.html. Zugegriffen: 17. Juni 2020.

Leucht, S. 2018. Sündenböcke und der Stoff, aus dem Legenden sind. Nachtkritik.de. https://nachtkritik.de/index.php?option=com_content\&view=article\&id=15240:der-mue nchner-kulturausschuss-debattiert-ueber-die-causa-lilienthal-und-das-ende-seiner-intend anz-am-den-kammerspielen\&catid=101\&Itemid=84. Zugegriffen: 17. Juni 2020.

Michaels, Bianca. 2021. Theatre Crisis, Local Farce, or Institutional Change? The Controversy Surrounding the Munich Kammerspiele 2018 from an Institutional Logics Perspective. In Theatre Institutions in Crisis: European Perspectives, Hrsg. Balme, C. und T. Fisher. London: Routledge (im Druck).

Noack, B. 2016. Das Theater muss seine Funktion neu definieren. Neue Zürcher Zeitung 12.Dezember 2016. https://www.nzz.ch/feuilleton/matthias-lilienthal-und-die-mue nchner-kammerspiele-theater-muss-seine-funktion-neu-definieren-ld.134076. Zugegriffen: 17. Juni 2020.

o. A. 2018. Demo für die Kammerspiele. Süddeutsche Zeitung 17. April 2018. https://www. sueddeutsche.de/muenchen/auf-dem-marienplatz-demo-fuer-die-kammerspiele-1.394 8282. Zugegriffen: 17. Juni 2020.

Quaas, R. 2018. Offener Brief an die SZ-Münchenkultur-Chefin zu ihrem Kommentar 'Wer hat eine Vision für München. Facebook 27. März 2018. https://www.facebook.com/Sta dtrat.Richard.Quaas/posts/1809452025778367?_xts_\%5B0\%5D=68.ARBIwzJIRvZI exj2X668kE0xQqi9C3k8LTyRwPP9p1qYqjmnXfxiTU8dtt_kBv_Ydj-g4fKtg5Be85v w2JFLYNV4tWPWDwhXMN9lyF1J5IhuTheqna4pQorJ7b0TNh4zjHe8qaaJOXf7KT g3X_0QAf0U0cF96CC8kbBoItU08iMr4yHXt33y0net-aX9hF7tbZsIP5xtELBXqq9L7 PybN9K58Y7CBXdRmumCHvrn7HWlO4o1fcOKghM-aYSllwXgeor-zNdxG0d6veYC nMbpV8myTGxy-A9a7K7NbrsflK1IsIIabe0vMKQeualU4sVERhEERvuJVp4R11kb 7hmXsA\&_tn_=K-R. Zugegriffen: 07. Sept. 2019.

Schleicher, Michael. 2018. Stadt will Lilienthal-Nachfolger bis Jahresende. Merkur. 13. April 2018. https://www.merkur.de/kultur/stadt-will-lilienthal-nachfolger-bis-jahresende9776388.html. Zugegriffen: 27. Dezember 2020.

Scott, W. Richard. 2014. Institutions and organizations: ideas, interests and identities. Stanford University: Sage Publications.

Stempel, Lukas. 2020. Mit der Stadtgesellschaft ins Spiel kommen - Der Fonds „Heimspiel“ der Kulturstiftung des Bundes. In diesem Band.

Von Cossel, Friederike. 2011. Entscheidungsfindung im Kulturbetrieb am Beispiel der Spielplangestaltung im Theater. München: Hampp.

Westle, Bettina. 2000. Legitimation. In Politik-Lexikon, Hrsg. E. Holtmann, 341-346. München: DeGruyter \& Oldenbourg. 
Open Access Dieses Kapitel wird unter der Creative Commons Namensnennung 4.0 International Lizenz (http://creativecommons.org/licenses/by/4.0/deed.de) veröffentlicht, welche die Nutzung, Vervielfältigung, Bearbeitung, Verbreitung und Wiedergabe in jeglichem Medium und Format erlaubt, sofern Sie den/die ursprünglichen Autor(en) und die Quelle ordnungsgemäß nennen, einen Link zur Creative Commons Lizenz beifügen und angeben, ob Änderungen vorgenommen wurden.

Die in diesem Kapitel enthaltenen Bilder und sonstiges Drittmaterial unterliegen ebenfalls der genannten Creative Commons Lizenz, sofern sich aus der Abbildungslegende nichts anderes ergibt. Sofern das betreffende Material nicht unter der genannten Creative Commons Lizenz steht und die betreffende Handlung nicht nach gesetzlichen Vorschriften erlaubt ist, ist für die oben aufgeführten Weiterverwendungen des Materials die Einwilligung des jeweiligen Rechteinhabers einzuholen.

(c) (1) 